\title{
The Study of Future Mars Immigration Policy Based on Analytic Hierarchy Process \\ Changkun Shao
}

School of North China Electric Power University, BaoDing 071003, China

18833213828@163.com

Keywords: policy, AHP, TTFA, TOPSIS.

Abstract. In this paper, we study the future society on Mars and build a model on three key factors which include equality, education and income to evaluate the society system and find an ideal point in the system. We study the evaluation criteria of social development. In order to obtain accurate data, we search in the www.census.gov where the data is authoritative. In the mean time, we try to define some parameters related to equality, education and income. And then we establish model 1 which can be used to evaluate the system of the future city on Mars by combining with Factor analysis method (TTFA) and Technique Order Preference Ideal Solution (TOPSIS).[1] And finally we obtain a evaluation model.

\section{Introduction}

Mars has a lot in common with the earth. Both having liquid water, atmosphere,

mountains, plains, deserts. With the increasing population and the worsening of the earth's environment, Immigrating to Mars may be one of the things that must be done in the future. So it is necessary for us to study ahead of time about the Mars immigration policy. We are also more interested in this.

\section{Future Mars Immigration Policy}

\subsection{Basial Assumptions}

Mars has the same social characteristic as the population on Earth

Short-term population dose not change

Mars social is stability

\subsection{Model: How to Evaluate an Utopia? (Figure 1)}

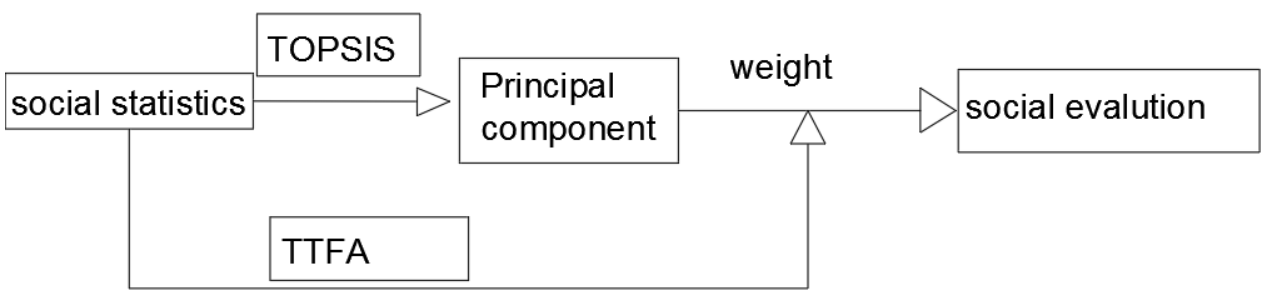

Figure 1 The evaluation method 
Table 1 Various levels of analytic hierarchy process

\begin{tabular}{|c|c|c|}
\hline $\begin{array}{l}\text { Parameter's } \\
\text { symbol }\end{array}$ & Explanation of parameter & Group \\
\hline $\mathbf{X 1}$ & Gini Coefficient( The gap between rich and poor society) & \multirow{2}{*}{ Equality(F1) } \\
\hline $\mathbf{X} 2$ & Gender Equality Index( A description of gender equity) & \\
\hline $\mathbf{X 3}$ & $\begin{array}{l}\text { Engel coefficient( The ratio of food expenditure of total } \\
\text { consumption expenditure) }\end{array}$ & \multirow{2}{*}{ Income(F2) } \\
\hline $\mathbf{X} 4$ & $\begin{array}{c}\text { Per Capita Disposable Income(Residents' discretionary } \\
\text { income) }\end{array}$ & \\
\hline $\mathbf{X 5}$ & $\begin{array}{l}\text { Average education years( The average number of years of } \\
\text { schooling) }\end{array}$ & \multirow[t]{2}{*}{ Education(F3) } \\
\hline X6 & Education spending accounted for GDP & \\
\hline
\end{tabular}

P.s: Other symbols instructions will be given in the text.

The purpose of equality is that made vulnerable groups such as female, children the future city in Mars have a fair living environment (Table 1).

The purpose of income is that made the minimum wage can satisfy minimum material needs of people and made the salary distribution optimal.

The purpose of education is that made people obtain the skills to satisfy needs of future.

We have gotten 6 parameters related to 3 critical factors. We wish to establish a scoring system to evaluate the system.

We use Technique QrderPreference IdealSolution(TOPSIS)(1981) to calculate weights of each parameter.

And then, we apply Factor analysis method (TTFA) with (Statistical Product and Service Solution) SPSS to find critical parameters.

So we can get the system of equation:

$$
x_{i}=\sum_{i=1}^{p} \sum_{j=1}^{k} a_{i j} \bullet f_{j}+e_{i} \quad(\mathrm{k}<\mathrm{p})
$$

Where $x i$ is the $i$-th parameter, aij represents the $j$-th factor's load to the $i$-th parameters, and the $i$-th special factor.

The system of equation can be expressed as a matrix and the expression is

$\mathrm{X}=\mathrm{AF}+\mathrm{e}$

Where $\mathrm{X}$ represents the matrix, A represents Factor load matrix, $\mathrm{F}$ is factor, e is special factor, which represents the part which can not be resolved by factors.

And then, we will build factor analysis model.

Firstly, we can get the equations:

$$
x_{i j}{ }^{\prime}=\frac{x_{i j}-\bar{x}_{j}}{\sqrt{S_{j}}} \quad x_{j}=\frac{\sum_{j=1}^{k} x_{i j}}{n} \quad S_{j}=\frac{\sum_{j=1}^{k} x_{i j}-\bar{x}_{j}}{n-1}
$$

Where $\mathrm{Sj}$ is standard deviation, $\overline{x_{j}}$ is the average of the $\mathrm{j}$-th factors.

We can get standardization matrix $\mathrm{R}$ and calculate simple correlation coefficient matrix $\mathrm{R}$. 
Next, we get the characteristic equation below:

$$
|R-\lambda E|=0
$$

Whose solutions are $\lambda_{i}, i=1,2, \ldots, p$. If $\lambda_{1} \geq \lambda_{2} \geq \lambda_{3} \geq \ldots \geq \lambda_{6} \geq 0$, we will obtain the number $p$ of factors based on cumulative variance contribution rate. The cumulative variance contribution rate the first $\mathrm{k}$ factors can be calculated by the below equation.

And then, we will calculate dispersion between initial factor and common factor. Carry on orthogonal factor rotation and oblique factor rotation with variance max method to obtain orthogonal or oblique factor load matrix. Here, we will get common factor.

Finally, we will calculate overall ratings and common factor ratings. The $\mathrm{j}$-th factor's contribution to the $\mathrm{i}$-th parameter is that:

$$
F_{j i}=\overline{W_{j 1}} X_{1 i}+\overline{W_{j 2}} X_{2 i}+\ldots+\overline{W_{j p}} X_{p i}
$$

Where $\overline{W_{j 1}}, \overline{W_{j 2}}, \ldots, \overline{W_{j p}}$ presents factor value parameters between the $\mathrm{j}$-th factor and the 1-th, the 2 -th,..., the p-th initial parameters. We can easily find that factor rating is weighted average of parameters.

Firstly, in order to investigate data of Gini Coefficient(x1), Gender Equality(x2), Engel coefficient(x3), Per Capita Disposable(x4), Average education years(x5), Education spending accounted for GDP in the past 10 years(x6), we search in ... website. Using historical data, we determine initial conditions for our model.[2]

Secondly, we obtain the weight of each parameter via TOPSIS. The table below is the weights we get:

Table 2 The weights of parameters

\begin{tabular}{|c|c|}
\hline Parameter & Weight \\
\hline $\mathrm{X} 1$ & 0.1209 \\
\hline $\mathrm{X} 2$ & 0.1512 \\
\hline $\mathrm{X} 3$ & 0.2230 \\
\hline $\mathrm{X} 4$ & 0.2134 \\
\hline $\mathrm{X} 5$ & 0.1710 \\
\hline $\mathrm{X} 6$ & 0.1206 \\
\hline
\end{tabular}

And then, we build a model which can be used to evaluate society with SPSS. We put the six parameters into SPSS.

Eigenvalue of each factor be presented in figure 2. In Figure 2, we summarize three main factor. The eigenvalue of these factors are higher than others obviously. [3]

According to the above data, we can get the system of Matrix equation:

$$
\left\{\begin{array}{l}
W_{1}=0.407 \\
W_{2}=0.314 \\
W_{3}=0.395
\end{array}\right.
$$

Finally, we can know that the weights of equality, income and education are $0.407,0.314,0.395$. 


\subsection{Strength and Weakness}

\subsubsection{Strength}

The fundamental strengths of our model are its robustness and flexibility. All the data is fully parameterized, so the model can be applied to evaluate the Utopia society and find the optimal point of the system.

In addition, our method is easy and, it is fast and shows an excellent stability.

\subsubsection{Weakness}

Due to the limit of the time, the data of the experiment is far from enough. There are certain subjective factors in our model, that is to say that our model may be affected by our subjective judgment. So our method has a certain deviation.

\section{Summary}

In this paper, we carry the research on Utopia on Mars by setting up AHP model. We can get the weights of equality, income and education. This provides guidance for future studies on the immigration policy of Mars. This study is of great significance.

\section{References}

[1]. Shixin Gong, Cheng Shao, Li Zhu, Energy Efficiency Evaluation based on DEA integrated Factor Analysis with respect to Operation Classification in Ethylene Production, Chinese Journal of Chemical Engineering, Available online 23 January 2017, ISSN 1004-9541.

[2]. Petra Radeljak Kaufmann, Integrating factor analysis and the Delphi method in scenario development: A case study of Dalmatia, Croatia, Applied Geography, Volume 71, June 2016, Pages 56-68, ISSN 0143-6228.

[3]. Adel Hatami-Marbini, Fatemeh Kangi, An extension of fuzzy TOPSIS for a group decision making with an application to tehran stock exchange, Applied Soft Computing, Volume 52, March 2017, Pages 1084-1097, ISSN 1568-4946. 